\title{
Correction to: Design and rationale of the non-interventional, edoxaban treatment in routiNe clinical prActice in patients with venous ThromboEmbolism in Europe (ETNA-VTE-Europe) study
}

Alexander T. Cohen ${ }^{1 *}$, Cihan Ay ${ }^{2}$, Philippe Hainaut ${ }^{3}$, Hervé Décousus ${ }^{4}$, Ulrich Hoffmann ${ }^{5}$, Sean Gaine ${ }^{6}$, Michiel Coppens ${ }^{7}$, Pedro Marques da Silva ${ }^{8}$, David Jimenez ${ }^{9}$, Beatrice Amann-Vesti ${ }^{10}$, Bernd Brüggenjürgen ${ }^{11}$, Pierre Levy ${ }^{12}$, Julio Lopez Bastida ${ }^{13}$, Eric Vicaut ${ }^{14}$, Petra Laeis ${ }^{15}$, Eva-Maria Fronk ${ }^{15}$, Wolfgang Zierhut ${ }^{15}$, Thomas Malzer ${ }^{15}$, Peter Bramlage ${ }^{16}$, Giancarlo Agnelli ${ }^{17}$ and on behalf of the ETNA-VTE-Europe investigators

\section{Correction to: Thrombosis Journal (2018) 16:9 DOI https://doi.org/10.1186/s12959-018-0163-7} Following publication of the original article [1], the authors reported that one of the authors' name was given incorrectly. In this Correction the incorrect and correct author name are shown. The original publication of this article has been corrected.

Originally the author name has been published as:

\begin{abstract}
Mancha, Talavera de la Reina, Toledo, Spain. ${ }^{14}$ Department of Medicine, Université Paris Descartes, Paris, France. ${ }^{15}$ Daiichi Sankyo Europe $\mathrm{GmbH}$, Munich, Germany. ${ }^{16}$ Institute for Pharmacology and Preventive Medicine, Berlin, Germany. ${ }^{17}$ Internal and Cardiovascular Medicine-Stroke Unit, University of Perugia, Perugia, Italy.
\end{abstract}

Published online: 31 May 2018

Reference

1. Cohen, et al. Thromb J. 2018;16:9. https://doi.org/10.1186/s12959-018-0163-7.

- David Jimenez Castro

The correct author name is:

- David Jimenez

\section{Author details}

'Guy's and St Thomas' NHS Foundation Trust, King's College London, London, UK. ${ }^{2}$ Clinical Division of Haematology and Haemostaseology, Department of Medicine I, Medical University of Vienna, Vienna, Austria. ${ }^{3}$ Department of General Internal Medicine, Cliniques Universitaires Saint Luc, UCL, Bruxelles, Belgium. ${ }^{4}$ Centre Hospitalier Universitaire de Saint-Etienne, Saint-Priest En Jarez, France. ${ }^{5}$ Division of Angiology, Medical Clinic IV, University Hospital, Ludwig-Maximilians-University, Munich, Germany. ${ }^{6}$ National Pulmonary Hypertension Unit, Mater Misericordiae University Hospital, Dublin, Ireland. ${ }^{7}$ Department of Vascular Medicine, Academic Medical Center, Amsterdam, The Netherlands. ${ }^{8}$ Department of Internal Medicine, Arterial Investigation Unit, Hospital de Santa Marta, Lisbon, Portugal. ${ }^{9}$ Respiratory Department, Ramón y Cajal Hospital, Madrid, Spain.

${ }^{10}$ Division of Angiology, University Hospital Zurich, Zurich, Switzerland.

${ }^{11}$ Institute for Health Economics, Steinbeis-University, Berlin, Germany.

${ }^{12}$ LEGOS, Université Paris - Dauphine, Paris, France. ${ }^{13}$ University of Castilla-La

\footnotetext{
* Correspondence: alexander.cohen@kcl.ac.uk

'Guy's and St Thomas' NHS Foundation Trust, King's College London, London, UK
}

(c) The Author(s). 2018 Open Access This article is distributed under the terms of the Creative Commons Attribution 4.0 International License (http://creativecommons.org/licenses/by/4.0/), which permits unrestricted use, distribution, and reproduction in any medium, provided you give appropriate credit to the original author(s) and the source, provide a link to the Creative Commons license, and indicate if changes were made. The Creative Commons Public Domain Dedication waiver (http://creativecommons.org/publicdomain/zero/1.0/) applies to the data made available in this article, unless otherwise stated. 